\title{
Basipodella barpacticola n.gen., n. sp. (Crustacea, Copepoda)
}

\author{
K.-H. BECKER $\dagger$ \\ Zoologisches Institut und Museum der Universität Kiel; Kiel, \\ und \\ Biologische Anstalt Helgoland; \\ Hamburg 50, Bundesrepublik Deutschland
}

\begin{abstract}
Basipodella barpacticola n. gen., n. sp. (Crustacea, Copepoda). Several stages of the copepod Basipodella barpacticola n. gen., n. sp., which lives as a parasite on harpacticoid copepods, are described on the basis of four individuals obtained from the Peruvian deep-sea. The taxonomic position of the new species is discussed.
\end{abstract}

\section{EINLEITUNG}

Es vergeht kein Jahr, in dem nicht mehrere neue Copepoda parasitica beschrieben werden. Die hier zu behandelnde Form nimmt jedoch in mancher Beziehung eine Sonderstellung ein.

Bei Untersuchungen über Tiefsee-Copepoden aus dem Peru-Graben wurden an einigen dieser Tiere, die alle verschiedenen Genera angehörten, verschiedene Stadien eines winzigen Krebses angeheftet gefunden. Die Größe der angehefteten Exemplare schwankt zwischen $85 \mu \mathrm{m}$ und etwas über $100 \mu \mathrm{m}$ ohne Eiersack. Die hier gefundene neue Art gehört zu den kleinsten bekannten Arthropoden. Dieser Umstand hat die Präparation und die lichtmikroskopische Untersuchungen sehr erschwert.

\section{DIAGNOSE}

$\mathrm{L}=85 \mu \mathrm{m} ; \mathrm{L}_{(\mathrm{eeph})}=37 \mu \mathrm{m} ; \mathrm{L}_{(\text {thor })}=35 \mu \mathrm{m} ; \mathrm{L}_{(\mathrm{abd})}=13 \mu \mathrm{m} ;$

$\mathrm{B}=31 \mu \mathrm{m}$ (maximal); $\mathrm{LBI}=36,5$. Alle Maße beziehen sich auf das einzige Exemplar ohne Eiersack (Abb. 1 A), welches auch Typus ist. Der Körper gliedert sich in drei Tagmata: den Cephalothorax, den aus 6 Segmenten bestehenden Thorax und das Abdomen, das 5 Segmente aufweist und eine Furca trägt.

Am Cephalothorax konnten folgende Strukturen festgestellt werden:

Nahe dem rostralen Rand inseriert dorsal ein pariger Anhang (a), der, wie Abbildung $2 \mathrm{~A}$ und $\mathrm{B}$ zeigen, wohl zur Anheftung an die Cuticula des Wirtes dient. Es mag sich dabei um die Antennulae (A. 1) handeln.

+ Dr. K.-H. BECKER, Mitglied der Taxonomischen Arbeitsgruppe an der Biologischen Anstalt Helgoland, verstarb während der Drucklegung dieser Arbeit am 27.4. 1975. 
Die folgende Struktur besteht aus einem cuticularisierten Ring, der die Funktion eines Saugnapfes besitzt und aus dessen Mitte ein stilettförmiger Dorn in das Innere des Wirtes ragt. Es ist denkbar, daß der Saugnapf der Antenna (A. 2) und das Stilett der Mandibel (Md.) entspricht (Abb. 2 A, a, b, c). Die übrigen beobachteten Extremitäten bestehen aus schlanken Dornen, die offenbar in Richtung des Saugnapfes verlaufen (Abb. $2 \mathrm{~A}, \mathrm{~d}, \mathrm{e}, \mathrm{f}$ ). Da ein Ablösen des Cephalothorax von der Cuticula des Wirtes ohne Verlust genannter Extremitäten nicht möglich ist, beschränken sich die Beobachtungen auf die abgebildete Lateralansicht. Ob es sich bei den Dornen um die Maxillen (Mx. 1, Mx. 2) und den Maxillipeden (Mxp.) handelt, ist mit Sicherheit nicht zu beweisen.

Die 6 Thoracalsegmente, von denen keines mit dem Cephalothorax verwachsen ist, tragen sämtlich ein wohl ausgeprägtes gleichartiges Paar Schwimmbeine. Der Protopodit ist besonders kräftig entwickelt und übt zur Hauptsache die Schwimmfunktion aus. Am Protopoditen inserieren je ein reduzierter eingliedriger Endo- und Exopodit, die je 2 relativ lange Borsten tragen (Abb. $1 \mathrm{C}$ ).

Deutlich abgesetzt gegen den Thorax schließt sich das kurze, aus 5 Segmenten bestehende Abdomen an. Die Furca ist etwa so lang wie breit und trägt 2 sichtbare terminale Borsten. Zu beachten ist die Artikulation des Abdomens hinter dem letzten Thoracalsegment.

Einen biologisch sehr interessanten Aspekt vermitteln die Darstellungen der $\mathrm{Ab}$ bildungen $2 \mathrm{~B}, \mathrm{C}$ und D. Hier werden gewissermaßen die Stadien einer Metamorphose von Basipodella wiedergegeben. Abbildung 2 B zeigt ein Stadium, in welchem die Gonaden Tergite und Sternite weit auseinander gedrängt haben und sich innerhalb eines „Sackes" ïber die Furca hinaus ausdehnen. Dorsal der Gonaden sind Eizellen zu beobachten. Die Beine und das Abdomen sind noch vorhanden.

Abbildung $2 \mathrm{C}$ gibt in etwa das eben beschriebene Stadium aus dorsaler Sicht wieder. Sichtbar sind die 6 Tergite, die biserial angeordneten Gonaden sowie der alles umhüllende Sack. Dieser geht offenbar im wesentlichen auf die Pleuralhäute zurück.

Abbildung $2 \mathrm{D}$ repräsentiert das letzte bisher beobachtete Stadium. Bis auf den Cephalothorax sind alle vorher erkennbaren Strukturen des Tieres verschwunden. Ubrig bleibt ein einfaches sackförmiges Gebilde, welches die Geschlechtsprodukte und Reservestoffe enthalten dürfte. Im abgebildeten Falle handelt es sich möglicherweise um ein Männchen, da Eizellen wie bei den Exemplaren der Abbildungen 2 B und C nicht zu erkennen sind. Das Tier bestünde dann aus einer einzigen Spermotophore. Denkbar ist aber auch, daß nur ein sehr dotterreiches Ei gebildet wird, wie ich es bei dem Tiefsee-Harpacticiden Pseudomesocbra minor BECKER, 1974 beobachten konnte. Letzterer Fall besitzt jedoch gerade für einen Parasiten die geringste Wahrscheinlichkeit.

\section{DISKUSSION}

Vorausgeschickt sei, daß der Verfasser Basipodella barpacticola auf Grund der Körpergliederung sowie der parasitischen Lebensweise als Copepoden betrachtet. Es bereitet allerdings einige Schwierigkeiten, die Art in das System der Copepoda einzuordnen, da bei der Kleinheit und bei den wenigen Exemplaren kein Homologiekriterium uneingeschränkt anwendbar ist. 
Während die Antennen und Mundwerkzeuge gemäß einer parasitischen Lebensweise umgewandelt sind, muß den 6 schwimmbeintragenden freien Thoracalsegmenten größte Aufmerksamkeit gewidmet werden.

Per definitionem gilt für die Unter-Klasse Copepoda, daß das 1. Thoracalsegment mit dem Kopf verschmolzen ist und als Extremität den Maxillipeden trägt. Darauf folgen 5 Beinpaare, von denen das letzte meist in irgendeiner Weise umgestaltet ist.

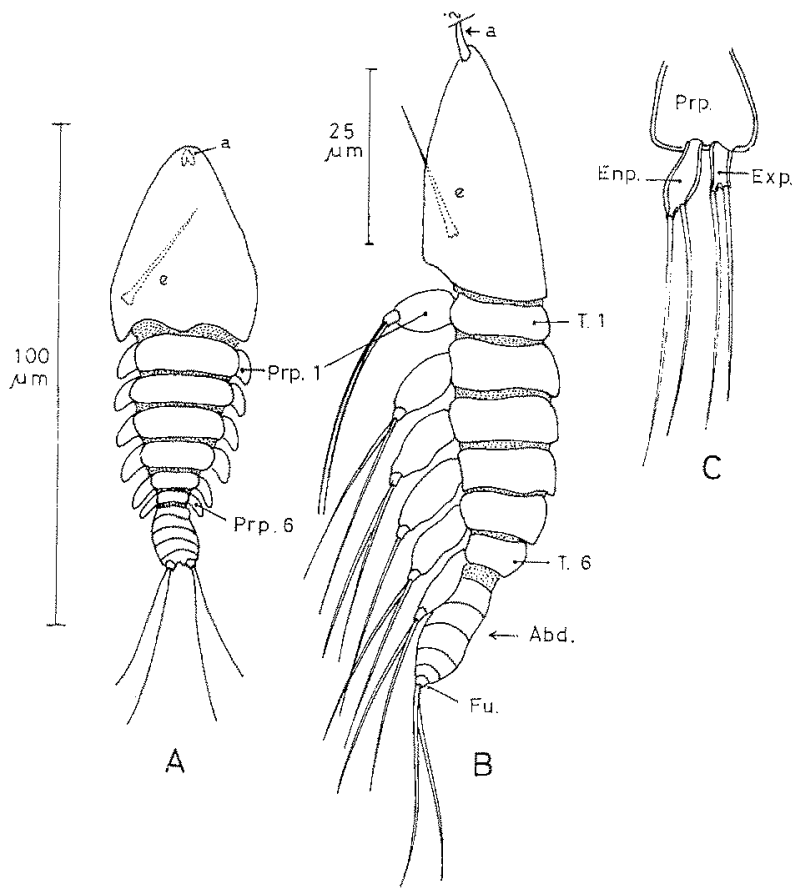

Abb. 1: Basipodella harpacticola. A Dorsalansicht; B Seitenansicht; C Thoracopod. a $=$ Antennula? $\mathrm{b}=$ Antenna? $\mathrm{c}=$ Mandibel ? $\mathrm{d}=$ Maxillula ? $\mathrm{e}=$ Maxilla? $\mathrm{f}=$ Maxilliped ? Abd. = Abdomen, Ceph. = Cephalothorax, Enp. = Endopodit, Exp. = Exopodit, Ez. = Eizellen, Fu. $=$ Furca, Int. $=$ Darm, Ov. = Ovarium, Prp. = Protopodit, P.1 $=$ P.6 - Pereiopoden $1-6, \mathrm{~T} .=$ Thoracomer, $\mathrm{LBI}=$ Längen-Breiten-Index

An diese 6 beintragenden Segmente schließt sich das Abdomen mit 5 Segmenten an, yon denen die ersten beiden bei $q$ q zum sogenannten Genitaldoppelsegment verschmelzen. Nun trägt aber bei Cyclopiden und Harpacticiden das erste Abdominalsegment ein weiteres, wenn auch stark reduziertes Extremitätenpaar (P. 6). Eine Art, Limnocletodes bebningi SMIRNov (Harpacticoidea), weist sogar Rudimente eines 7. und 8. Beinpaares auf.

Die hier aufgeworfene Problematik kann in diesem knappen Rahmen unmöglich abgehandelt werden. Sie im Auge zu behalten, ist aber für die Deutung der morphologischen Verhältnisse bei Basipodella barpacticola von Wichtigkeit. Das Vorhandensein von 6 freien, mit Schwimmbeinen versehenen Segmenten läßt im Rahmen der Copepoda nämlich nur zwei Deutungen zu: 


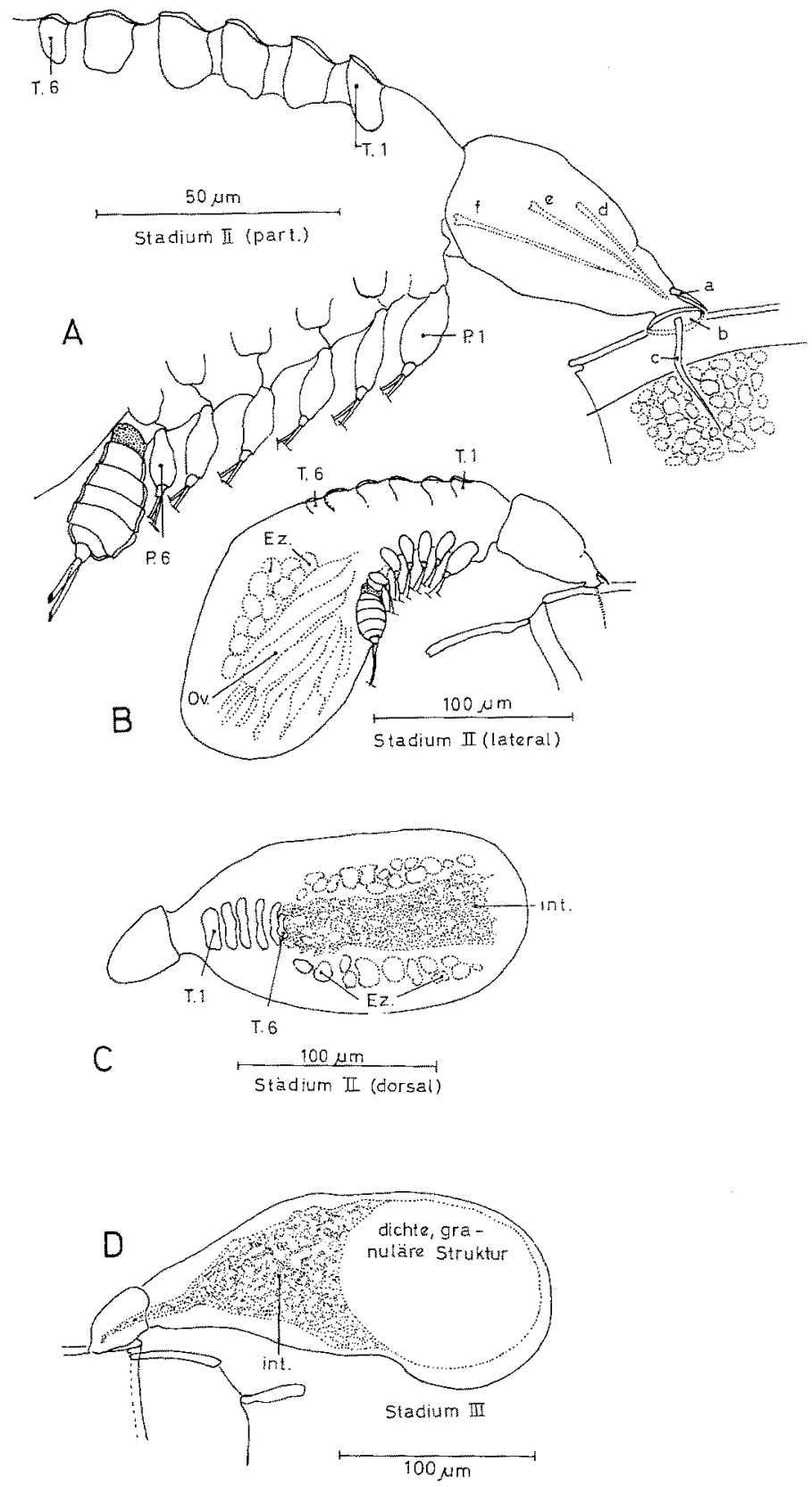

Abb. 2: Basipodella barpacticola. A Stadium II (partiell); B Stadium II (lateral); C Stadium II (dorsal); D Stadium III. (Abkürzungen vgl. Legende zu Abb. 1) 
(1) Geht man von den oben geschilderten Verhältnissen aus und setzt gleichzeitig voraus, daß Basipodella ein mit dem Kopf verschmolzenes 1. Thoracalsegment besitzt, so ergibt sich, daß die neue Form 6 freie Thoracomeren + ein in den Cephalothorax einbezogenes, also im ganzen 7 Thoraxsegmente aufweist. Diese Folgerung wäre mit den Gegebenheiten bei Cyclopiden und Harpacticiden vereinbar und würde gleichzeitig bedeuten, daß die weibliche Genitalöffnung bei den genannten Unterordnungen am 7 . Thoracomer lokalisiert ist.

(2) Lehnt man von vornherein das Vorhandensein eines 7. Thoracopoden (P.6) bei Copepoden ab, was zweifellos schwer zu begründen wäre und eine Diskussion über reduzierte Extremitäten nach sich zöge, so bleibt im Hinblick auf Basipodella nur folgender Schluß übrig: Das 1. Thoracomer ist nicht mit dem Kopf verschmolzen und Basipodella harpacticola besitzt somit keinen Cephalothorax, sondern einen Kopf, was bei Crustaceen recht selten ist. Damit wäre eine Einordnung der Art in die Unterklasse Copepoda nicht möglich.

Trotz des Fehlens einwandfreier Homologisierungsmöglichkeiten, zumindest zur Zeit, beinhaltet nach Ansicht des Verfassers die erste Deutung den größeren Wahrscheinlichkeitsgehalt. Es bedarf allerdings noch weiterer Befunde, um zu einer gesicherten phylogenetischen Aussage zu gelangen.

So ist das Hauptanliegen dieser Publikation, das Interesse auf diese zweifellos sehr ursprüngliche Crustaceen-Gruppe, wie sie Basipodella repräsentiert, zu lenken.

\section{ZUSAMMENFASSUNG}

1. Verschiedene Stadien von Basipodella barpacticola n. gen., n. sp., eines an Harpacticiden parasitierenden Copepoden, werden an Hand von vier Exemplaren, die aus der peruanischen Tiefsee stammen, beschrieben.

2. Die Stellung der neuen Art im System der Copepoda wird vor allem im Hinblick auf das Vorhandensein von sechs freien, mit Schwimmbeinen ausgestatteten Segmenten diskutiert.

\section{ZITIERTE LITERATUR}

BECKER, K.-H., 1974. Eidonomie und Taxonomie abyssaler Harpacticoidea (Crustacea, Copepoda). Teil I: Cerviniidae - Ameiridae. Meteor Forsch.Ergebn. (D) 18, 1-28.

KaESTNER, A., 1967. Lehrbuch der speziellen Zoologie. Fischer, Stuttgart, 1 (2), 849-1242.

LANG, K., 1948. Monographie der Harpacticiden. Ohlsen, Lund, 1-2, 1-1683.

SARs, G. O., 1903. An account of the Crustacea of Norway. Bergen Mus., Bergen, 4, 1-171.

- 1911. An account of the Crustacea of Norway. Bergen Mus., Bergen, 5, 1-499.

- 1918. An account of the Crustacea of Norway. Bergen Mus., Bergen, 6, 1-225.

- 1921. An account of the Crustacea of Norway. Bergen Mus., Bergen, 7, 1-121.

Sonderdruckanforderungen an:

Zoologisches Institut und Museum

D-23 Kiel

Hegew ischstr. 3

Bundesrepublik Deutschland 